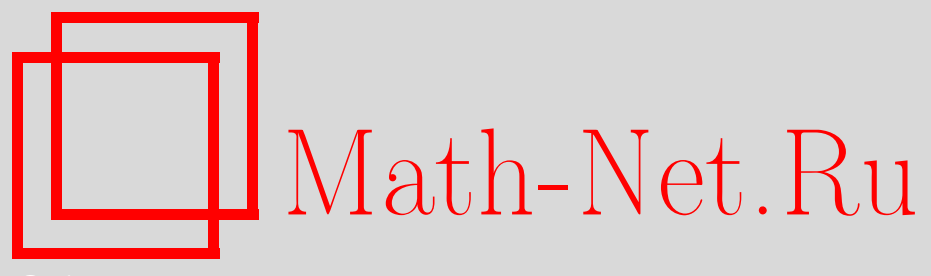

Е. А. Кудрявцева, Спиральность - единственный инвариант несжимаемых течений с непрерывной в $C^{1}$ топологии производной, Матем. заметки, 2016, том 99, выпуск 4, 626-630

DOI: https://doi.org/10.4213/mzm11061

Использование Общероссийского математического портала Math-Net.Ru подразумевает, что вы прочитали и согласны с пользовательским соглашением http: //www. mathnet.ru/rus/agreement

Параметры загрузки:

IP: 54.89 .56 .158

26 апреля 2023 г., 09:03:41

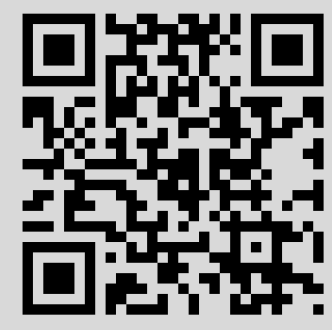




\section{Спиральность - единственный инвариант несжимаемых течений с непрерывной в $C^{1}$-топологии производной}

\section{Е. А. Кудрявцева}

Пусть $Q$ - гладкое компактное ориентированное 3-мерное многообразие с гладким краем $\partial Q$. Пусть $\mathcal{B}$ - множество точных 2-форм $B \in \Omega^{2}(Q)$ таких, что

$$
j_{\partial Q}^{*} B=0,
$$

где $j_{\partial Q}: \partial Q \rightarrow Q$ - отображение включения. Группа $\mathcal{D}=\operatorname{Diff}_{0}(Q)$ изотопных тождественному диффеоморфизмов $Q$ действует на множестве $\mathcal{B}$ в виде $\mathcal{D} \times \mathcal{B} \rightarrow \mathcal{B},(h, B) \mapsto h^{*} B$. Пусть $\mathcal{B}^{\circ}$ - множество 2-форм $B \in \mathcal{B}$ без нулей. Мы доказываем, что любой $\mathcal{D}$-инвариантный функционал $I: \mathcal{B}^{\circ} \rightarrow \mathbb{R}$, имеющий регулярную и непрерывную относительно $C^{1}$-топологии производную, локально на $\mathcal{B}^{\circ}$ (а в случае $Q=M \times S^{1}$ с $\partial Q \neq \varnothing$ - глобально на множестве 2-форм $B \in \mathcal{B}^{\circ}$, допускающих секущую поверхность, изотопную $\left.M \times\{*\}\right)$ выражается через спиральность.

1. Примеры $\mathcal{D}$-инвариантных функционалов на множестве $\mathcal{B}$ точных несжимаемых течений. Определим поток и спиральность.

ОПРедЕЛЕНИЕ 1. (А) Пусть $\Pi \in C_{2}(Q)$ - 2-цепь в $Q$ с границей $\partial \Pi \in C_{1}(\partial Q)$. Пусть $\llbracket \Pi \rrbracket \in \varkappa$ - проекция относительного класса гомологий $[\Pi] \in H_{2}(Q, \partial Q ; \mathbb{Q})$ в факторпространство

$$
\varkappa:=H_{2}(Q, \partial Q ; \mathbb{Q}) / \operatorname{Im} p_{*} \cong \operatorname{ker}\left(j_{\partial Q}\right)_{*}=\partial(\varkappa),
$$

где $p_{*}: H_{2}(Q ; \mathbb{Q}) \rightarrow H_{2}(Q, \partial Q ; \mathbb{Q}),\left(j_{\partial Q}\right)_{*}: H_{1}(\partial Q ; \mathbb{Q}) \rightarrow H_{1}(Q ; \mathbb{Q})$. Функционал

$$
\text { Flux }: \mathcal{B} \rightarrow \operatorname{Hom}_{\mathbb{Q}}(\varkappa, \mathbb{R}), \quad \operatorname{Flux}(B) \llbracket \Pi \rrbracket:=\int_{\Pi} B,
$$

назовем потоком на $\mathcal{B}$.

(В) Пусть $\varkappa^{\perp} \subseteq H_{1}(\partial Q ; \mathbb{Q})$ - произвольное векторное подпространство такое, что

$$
H_{1}(\partial Q ; \mathbb{Q})=\varkappa^{\perp} \oplus \operatorname{ker}\left(j_{\partial Q}\right)_{*} .
$$

Спиральностью на множестве $\mathcal{B}$ относительно подпространства $\varkappa^{\perp}$ назовем функционал

$$
\mathcal{H}_{\varkappa^{\perp}}: \mathcal{B} \rightarrow \mathbb{R}, \quad \mathcal{H}_{\varkappa^{\perp}}(B):=\int_{Q} B \wedge A, \quad B \in \mathcal{B}
$$

где $A \in \Omega^{1}(Q)$ - произвольная 1-форма на $Q$ такая, что $d A=B$ и $\oint_{\gamma} A=0$ для любой петли $\gamma: S^{1} \rightarrow \partial Q$ гомологического класса $[\gamma] \in \varkappa^{\perp}$.

Легко показать, что Flux и $\mathcal{H}_{\varkappa}$ определены корректно и $\mathcal{D}$-инвариантны.

Пример 1. Пусть $Q=M \times S^{1}$, где $M$ - компактная гладкая ориентированная поверхность с непустым гладким краем. Тогда на $M$ существует точная положительная форма площади $\omega \in \Omega^{2}(M)$. Пусть $B \in \mathcal{B}^{\circ}$ и $j_{M \times\{0\}}^{*} B=\omega$ (т.е. $M \times\{0\}$ является секущей поверхностью для 2-формы $B), S_{1}, \ldots, S_{d} \subseteq \partial M$ - граничные окружности, $\pi_{M}: Q \rightarrow M$ и $\pi_{S^{1}}: Q \rightarrow$

Работа выполнена при поддержке Российского фонда фундаментальных исследований (грант № 15-01-06302-а), и программы "Ведущие научные школы" (грант № НШ-7962.2016.1).

DOI: $10.4213 / \mathrm{mzm} 11061$ 
$S^{1}=\mathbb{R} / \mathbb{Z}$ - проекции. Так как $B$ точна, то в силу [1], [2] существуют диффеоморфизм $\psi \in \mathcal{D}$ и функция $H \in C^{\infty}(Q)$ такие, что

$$
\begin{gathered}
B=\psi^{*} B_{\omega, H},\left.\quad H\right|_{S_{1} \times S^{1}} \equiv 0, \\
\left.H\right|_{S_{i} \times\{t\}}=\mathrm{const}=: h_{i}(t) \quad \text { при } \quad t \in S^{1}, \quad i \in\{2, \ldots, d\},
\end{gathered}
$$

где

$$
B_{\omega, H}:=\pi_{M}^{*} \omega-d H \wedge d \pi_{S^{1}} .
$$

Для $i \in\{1, \ldots, d\}$ фиксируем точку $x_{i} \in S_{i}$ и пути $\gamma_{i k} \subset M$ из $x_{i}$ в точки $x_{k}, k \neq i$. Положим $\Pi_{i k}:=\gamma_{i k} \times S^{1}$. Поток и спиральность 2-формы $B$ таковы:

(А) классы $\llbracket M \times\{0\} \rrbracket, \llbracket \Pi_{12} \rrbracket, \ldots, \llbracket \Pi_{1 d} \rrbracket \in \varkappa$ образуют базис в $\varkappa$ и поток

$$
\operatorname{Flux}(B) \llbracket M \times\{0\} \rrbracket=\int_{M} \omega, \quad \operatorname{Flux}(B) \llbracket \Pi_{\ell k} \rrbracket=\int_{S^{1}} h_{\ell}(t) d t-\int_{S^{1}} h_{k}(t) d t ;
$$

(В) для любых $\ell, k \in\{1, \ldots, d\}$ возьмем в качестве $\varkappa^{\perp}$ подпространство $\varkappa_{\ell k}^{\perp} \subseteq H_{1}(\partial Q ; \mathbb{Q})$, порожденное гомологическими классами петель $\gamma_{k}:=\left\{x_{k}\right\} \times S^{1}$ и $S_{i} \times\{0\}, i \in$ $\{1, \ldots, d\} \backslash\{\ell\} ;$ по формуле Стокса спиральность

$$
\begin{aligned}
\mathcal{H}_{\varkappa_{11}^{\perp}}(B) & =-2 \int_{Q} H\left(\pi_{M}^{*} \omega\right) \wedge\left(d \pi_{S^{1}}\right)=-2 \operatorname{Cal}_{\omega}(\widetilde{\varphi}), \\
\mathcal{H}_{\varkappa_{\ell k}^{\perp}}(B)-\mathcal{H}_{\varkappa_{\ell \ell}^{\perp}}(B) & =\frac{1}{2}\left(\mathcal{H}_{\varkappa_{\ell \ell}^{\perp}}(B)-\mathcal{H}_{\varkappa_{k k}^{\perp}}(B)\right)=\operatorname{Flux}(B) \llbracket M \times\{0\} \rrbracket \operatorname{Flux}(B) \llbracket \Pi_{\ell k} \rrbracket
\end{aligned}
$$

при $k \neq \ell$. Здесь $\mathrm{Cal}_{\omega}: \widetilde{\mathcal{G}}_{\omega} \rightarrow \mathbb{R}-$ гомоморфизм Калаби [3], [4], $\widetilde{\mathcal{G}}_{\omega}-$ универсальная накрывающая группы $\mathcal{G}_{\omega}=S \operatorname{Diff}(M, \omega)$ симплектоморфизмов поверхности $(M, \omega)$ с $C^{\infty}$-топологией, элемент $\widetilde{\varphi} \in \widetilde{\mathcal{G}}_{\omega}$ отвечает 2-форме (3).

2. Дифференцируемые функционалы на $\mathcal{B}^{\prime \prime} \subseteq \mathcal{B}$ и $\left.\mathcal{H}_{\varkappa \perp}\right|_{\mathcal{B}^{\prime \prime}}$-связные подмножества $\mathcal{B}^{\prime \prime \prime} \subseteq \mathcal{B}^{\prime \prime}$. Обозначим через $\mathcal{A}$ (соответственно $\left.\mathcal{A}_{\varkappa \perp}\right)$ множество таких 1 -форм $A \in$ $\Omega^{1}(Q)$, что для любой петли $\gamma: S^{1} \rightarrow \partial Q$ (соответственно любой петли гомологического класса $[\gamma] \in \varkappa^{\perp}$, определение $\left.1(\mathrm{~B})\right)$ верно $\oint_{\gamma} A=0$. Пусть $\Phi \in \operatorname{Hom}_{\mathbb{Q}}(\varkappa, \mathbb{R})$ - линейная функция на (1) и

$$
\text { либо } \quad \mathcal{B}^{\prime}:=\operatorname{Flux}^{-1}(\Phi), \quad \mathcal{A}^{\prime}:=\mathcal{A}, \quad \text { либо } \quad \mathcal{B}^{\prime}:=\mathcal{B}, \quad \mathcal{A}^{\prime}:=\mathcal{A}_{\varkappa^{\perp}} .
$$

Ясно, что $\mathcal{B}^{\prime}$ и $\mathcal{A}^{\prime}$ являются $\mathcal{D}$-инвариантными, и для любого $B \in \mathcal{B}^{\prime}$ множество $\mathcal{B}^{\prime}-B$ является $C^{0}$-открытым в векторном пространстве $d \mathcal{A}^{\prime}$.

Пусть $\mathcal{B}^{\prime \prime} \subseteq \mathcal{B}^{\prime}$ есть $C^{1}$-открытое подмножество (например $\mathcal{B}^{\prime \prime}=\mathcal{B}^{\prime} \cap \mathcal{B}^{\circ}$ ).

ОПределение 2. Функционал $I: \mathcal{B}^{\prime \prime} \rightarrow \mathbb{R}$ назовем дифферениируемым в точке $B \in \mathcal{B}^{\prime \prime}$, если существует линейный функционал $D_{B} I: \mathcal{A}^{\prime} \rightarrow \mathbb{R}$, называемый производной функционала $I$ в точке $B$, такой, что для любого гладкого семейства 1 -форм $A_{u} \in \mathcal{A}^{\prime},-\varepsilon<u<\varepsilon$, со свойством $d A_{0}=0$

$$
\left.\frac{d}{d u}\right|_{u=0} I\left(B+d A_{u}\right)=D_{B} I\left(\left.\frac{d}{d u}\right|_{t=0} A_{u}\right) .
$$

Производную $D_{B} I$ назовем регулярной, если она является регулярным элементом двойственного пространства $\left(\mathcal{A}^{\prime}\right)^{*}$, т.е.

$$
D_{B} I\left(A^{\prime}\right)=\int_{Q} K_{I}(B) \wedge A^{\prime}, \quad A^{\prime} \in \mathcal{A}^{\prime}
$$

для некоторой измеримой 2-формы $K_{I}(B)$ на $Q$, называемой плотностъю функционала $D_{B} I$. Производную назовем $C^{k}$-непрерывной на $\mathcal{B}^{\prime \prime}$ (для $k \geqslant 0$ ), если $I$ дифференцируем всюду на $\mathcal{B}^{\prime \prime}$ и имеет регулярную производную, плотность которой есть непрерывное отображение $K_{I}: \mathcal{B}^{\prime \prime} \rightarrow \Gamma^{0}\left(\Lambda^{2} T^{*} Q\right)$ в смысле $C^{k}$-топологии на $\mathcal{B}^{\prime \prime}$ и $C^{0}$-топологии на $\Gamma^{0}\left(\Lambda^{2} T^{*} Q\right)$. 
Пример 2. Спиральность (2) дифференцируема всюду на $\mathcal{B}$ (а потому и на $\mathcal{B}^{\prime} \subseteq \mathcal{B}$ ). Ее производная регулярна и имеет плотность $K_{\mathcal{H}_{\varkappa}}(B)=B$. Поэтому ее производная непрерывна относительно любой топологии на $\mathcal{B}$.

ОПределение 3 . Подмножество $\mathcal{B}^{\prime \prime \prime} \subseteq \mathcal{B}^{\prime \prime}$ назовем $\left.\mathcal{H}_{\varkappa \perp}\right|_{\mathcal{B}^{\prime \prime}}$-связнъм, если любую пару его элементов с одним и тем же значением $c \in \mathbb{R}$ спиральности $\mathcal{H}_{\varkappa} \perp$ можно соединить кусочно-гладким путем в $\mathcal{H}_{\varkappa \perp}^{-1}(c) \cap \mathcal{B}^{\prime \prime}$.

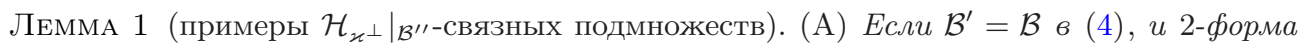
$B \in \mathcal{B}^{\prime \prime}$ такова, что $\mathcal{H}_{\varkappa^{\perp}}(B) \neq 0$, то любая достаточно малая $C^{1}$-окрестность $\mathcal{B}^{\prime \prime \prime} 2$-фбормиь В в $\mathcal{B}^{\prime \prime}$ является $\left.\mathcal{H}_{\varkappa \perp}\right|_{\mathcal{B}^{\prime \prime}}$-связной.

(В) Пусть $Q=M \times S^{1}$ как в примере 1. Пусть (4) и множество $\mathcal{B}^{\prime \prime} \subseteq \mathcal{B}^{\prime} \cap \mathcal{B}^{\circ}$ coстоит из всех 2-форм $\psi^{*} B_{\omega, H}$, где $\psi \in \mathcal{D}$ и $B_{\omega, H} \in \mathcal{B}^{\prime}$ как в (3). Тогда само множество $\mathcal{B}^{\prime \prime}=: \mathcal{B}^{\prime \prime \prime}$ является $\left.\mathcal{H}_{\varkappa \perp}\right|_{\mathcal{B}^{\prime \prime}}-$ связным.

Доказательство. (А) Любую пару $B_{0}, B_{1} \in \mathcal{H}_{\varkappa \perp}^{-1}(c) \cap \mathcal{B}^{\prime \prime \prime}$ соединим путем

$$
\left\{\left((1-u) B_{0}+u B_{1}\right) \sqrt{c / \mathcal{H}_{\varkappa}\left((1-u) B_{0}+u B_{1}\right)}\right\}_{u \in[0,1]}
$$

в $\mathcal{H}_{\varkappa \perp}^{-1}(c)$. Этот путь содержится в $\mathcal{B}^{\prime \prime}$ ввиду $C^{1}$-открытости $\mathcal{B}^{\prime \prime}$ и $C^{0}$-непрерывности $\mathcal{H}_{\varkappa}$.

(В) Множество $\mathcal{B}^{\prime \prime} C^{0}$-открыто (а потому и $C^{1}$-открыто) в $\mathcal{B}^{\prime}$. Ввиду [1] любая пара 2-форм из $\mathcal{H}_{\varkappa^{\perp}}^{-1}(c) \cap \mathcal{B}^{\prime \prime}$ имеет вид

$$
\psi_{0}^{*} B_{\omega, H_{0}}, \quad \psi_{1}^{*} B_{\lambda \omega, H_{1}}
$$

для некоторых $\psi_{0}, \psi_{1} \in \mathcal{D}, H_{0}, H_{1} \in C^{\infty}(Q), \lambda \in \mathbb{R}_{>0}$ и положительной формы площади $\omega \in$ $\Omega^{2}(M)$ таких, что $B_{\omega, H_{0}}, B_{\lambda \omega, H_{1}} \in \mathcal{H}_{\varkappa \perp}^{-1}(c)$. Так как любую пару $\psi_{0}, \psi_{1} \in \mathcal{D}$ можно соединить кусочно-гладким путем $\left\{\psi_{u}\right\}_{u \in[0,1]}$ В группе $\mathcal{D}[1]$, получаем путь $\left\{\psi_{u}^{*} B_{(1-u+u \lambda) \omega, a(u) H_{0}+u H_{1}}\right\}$ в $\mathcal{H}_{\varkappa^{\perp}}^{-1}(c) \cap \mathcal{B}^{\prime \prime}$, соединяющий 2-формы $\psi_{0}^{*} B_{\omega, H_{0}}$ и $\psi_{1}^{*} B_{\lambda \omega, H_{1}}$, где

$$
a(u):=\frac{1}{1-u+u \lambda}-\frac{u}{\lambda}, \quad 0 \leqslant u \leqslant 1 .
$$

3. Основной результат. Наш результат был анонсирован в [5] и аналогичен результатам [4], [6]. Мы его доказываем методом из работы [4].

Teорема. Пусть $\varkappa, \varkappa^{\perp}$ и $\mathcal{B}^{\prime} \kappa a \kappa$ в (1), (2) и (4). Пустъ $\mathcal{D}$-инвариантный функиионал $I: \mathcal{B}^{\prime} \rightarrow \mathbb{R}$ дифферениируем на $C^{1}$-открытом подмножестве $\mathcal{B}^{\prime \prime} \subseteq \mathcal{B}^{\prime} \cap \mathcal{B}^{\circ}$ и имеет регулярную и непрерывную относительно $C^{1}$-топологии производную на $\mathcal{B}^{\prime \prime}$. На любом

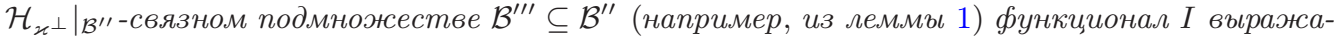
ется через спиральность, т.е. $I_{\mathcal{B}^{\prime \prime \prime}}=\left.h \circ \mathcal{H}_{\varkappa^{\perp}}\right|_{\mathcal{B}^{\prime \prime \prime}}$ для некоторой функции $h: \mathbb{R} \rightarrow \mathbb{R}$.

Лемма 2. Пусть $I: \mathcal{B}^{\prime} \rightarrow \mathbb{R}-\mathcal{D}$-инвариантный функционал, дифферениируемый в точке $B \in \mathcal{B}^{\prime \prime}$. Тогда для любых 1-формы $A^{\prime} \in \mathcal{A}^{\prime}$ и дифбеоморфизма $\psi \in \mathcal{D}$ с $\psi^{*} B=B$ верно равенство

$$
D_{B} I\left(A^{\prime}\right)=D_{B} I\left(\psi^{*} A^{\prime}\right) .
$$

ДокАзАтЕльство. В обозначениях определения 2 имеем

$$
I\left(B+d A_{t}\right)=I\left(B+\psi^{*} d A_{t}\right)=I\left(B+d \widetilde{A}_{t}\right),
$$

где $\widetilde{A}_{t}:=\psi^{*} A_{t}$, и $d \widetilde{A}_{0}=0$. Дифференцируя полученное соотношение

$$
I\left(B+d A_{t}\right)=I\left(B+d \widetilde{A}_{t}\right)
$$

по $t$ при $t=0$, получаем требуемое равенство для $A^{\prime}=\left.(d / d t)\right|_{t=0} A_{t}$. Лемма доказана. 
Следствие. Предположим, что в условиях леммы 2 производная $D_{B} I$ в точке $B$ регулярна и ее плотность $K_{I}(B)$ является непрерывной 2-формой на $Q$. Тогда

$$
K_{I}(B)=\psi^{*} K_{I}(B) .
$$

Если 2-форма $B$ не имеет нулей $\left(\right.$ m.е. $\left.B \in \mathcal{B}^{\circ}\right)$, то $K_{I}(B)=\lambda_{I}(B) B$ для некоторой функиии $\lambda_{I}(B) \in C(Q)$, постоянной на любой интегральной кривой поля ядер 2-формы $B$.

ДокАЗАтЕЛЬСтво. Равенство из леммы 2 перепишем в виде

$$
\int_{Q} K_{I}(B) \wedge A^{\prime}=\int_{Q} K_{I}(B) \wedge \psi^{*} A^{\prime} .
$$

Но левая часть этого равенства равна $\int_{Q}\left(\psi^{*} K_{I}(B)\right) \wedge \psi^{*} A^{\prime}$. Поэтому

$$
\int_{Q}\left(\psi^{*} K_{I}(B)-K_{I}(B)\right) \wedge \psi^{*} A^{\prime}=0 .
$$

Так как 1-форма $A^{\prime} \in \mathcal{A}^{\prime}$ любая (и, в частности, можно взять $A^{\prime} \in \mathcal{A} \subseteq \mathcal{A}^{\prime}$ с носителем в сколь угодно малой окрестности любой внутренней точки из $Q)$, а 2-форма $K_{I}(B)$ непрерывна на $Q$, получаем первое требуемое равенство $K_{I}(B)=\psi^{*} K_{I}(B)$.

Если $B$ не имеет нулей, то любая точка из $Q \backslash \partial Q$ имеет окрестность $U \approx(-\varepsilon, \varepsilon)^{3}$ в $Q \backslash \partial Q$ с регулярными координатами $w=(x, y, z) \in(-\varepsilon, \varepsilon)^{3}$ такими, что $\left.B\right|_{U}=d x \wedge d y$. По доказанному 2-форма

$$
\left.K_{I}(B)\right|_{U}=: L(w) d y \wedge d z+M(w) d z \wedge d x+N(w) d x \wedge d y,
$$

где $L, M, N \in C\left((-\varepsilon, \varepsilon)^{3}\right)$, инвариантна относительно преобразований $\psi: w \mapsto \widetilde{w}:=w+(0,0$, $f(w))$ для функций $f \in C^{\infty}\left((-\varepsilon, \varepsilon)^{3}\right)$ с компактным носителем и $\partial f / \partial z>-1$. Заметим, что

$$
\begin{aligned}
\left.\left(\psi^{*} K_{I}(B)-K_{I}(B)\right)\right|_{U}=( & L(\widetilde{w})-L(w)) d y \wedge d z+(M(\widetilde{w})-M(w)) d z \wedge d x \\
& +(N(\widetilde{w})-N(w)) d x \wedge d y+(L(\widetilde{w}) d y-M(\widetilde{w}) d x) \wedge d f(w) .
\end{aligned}
$$

Для любой точки $w_{0}=\left(x_{0}, y_{0}, z_{0}\right) \in(-\varepsilon, \varepsilon)^{3}$ рассмотрим такую функцию $f_{1}$, что $f_{1}(w)=$ $x-x_{0}$ в окрестности точки $w_{0}$. Ввиду $f_{1}\left(w_{0}\right)=0$ имеем

$$
0=\left.\left(\psi^{*} K_{I}(B)-K_{I}(B)\right)\right|_{w=w_{0}}=L\left(w_{0}\right) d y \wedge d x,
$$

откуда $L\left(w_{0}\right)=0$. Аналогично показывается, что $M\left(w_{0}\right)=0$. Поэтому $L \equiv M \equiv 0$ и $\left.K_{I}(B)\right|_{U}=\left.N(w) B\right|_{U}$.

Итак, соотношение $K_{I}(B)=\lambda_{I}(B) B$ доказано на $Q \backslash \partial Q$. Значит, оно верно и на $Q$, так как $B$ не имеет нулей. Так как

$$
0=\left.\left(\psi^{*} K_{I}(B)-K_{I}(B)\right)\right|_{U}=\left.(N(\widetilde{w})-N(w)) B\right|_{U}
$$

и $f$ любая, то функция $\left.\lambda_{I}(B)\right|_{U}=N(x, y, z)$ не зависит от $z$, т.е. постоянна на интегральных кривых поля $\operatorname{ker} B$. Следствие доказано.

ДоказатеЛЬСтво теОремЫ. Покажем, что для любой 2-формы $B \in \mathcal{B}^{\prime \prime}$ функция $\lambda_{I}(B) \in C(Q)$ из следствия постоянная на $Q$. Напомним, что векторное поле называется топологически транзитивным, если оно имеет всюду плотную интегральную кривую. Пусть $\mu$ - форма объема на $Q$. Согласно результату [7] векторное поле $\bar{B}$ такое, что $i_{\bar{B}} \mu=B$, можно $C^{1}$-аппроксимировать последовательностью $\left\{\bar{B}_{n}\right\}$ топологически транзитивных бездивергентных $C^{1}$-векторных полей на $Q$, касающихся $\partial Q$. Так как $\bar{B}$ точно, из доказательства в [7] следует, что $\bar{B}_{n}$ можно выбрать точным и имеющим поток $\operatorname{Flux}(B)$. Такую 2-форму $B_{n}$ можно $C^{1}$-аппроксимировать гладкой 2-формой $B_{n}^{s} \in \mathcal{B}^{\prime}$. Из $C^{1}$-открытости $\mathcal{B}^{\prime \prime}$ в $\mathcal{B}^{\prime}$ имеем $B_{n}^{s} \in \mathcal{B}^{\prime \prime}$. 
Итак, для любой пары точек $w, w^{\prime} \in Q$ существуют последовательности $\left\{B_{n}^{s}\right\}$ в $\mathcal{B}^{\prime \prime}$ и $\left\{w_{n}\right\},\left\{w_{n}^{\prime}\right\}$ в $Q$ такие, что $B_{n}^{s} \rightarrow B$ в $C^{1}$-топологии, $w_{n} \rightarrow w, w_{n}^{\prime} \rightarrow w^{\prime}$ и любая пара $w_{n}, w_{n}^{\prime}$ содержится в интегральной кривой поля $\bar{B}_{n}^{s}$. Значит,

$$
\lambda_{I}\left(B_{n}^{s}\right)\left(w_{n}\right)=\lambda_{I}\left(B_{n}^{s}\right)\left(w_{n}^{\prime}\right) .
$$

В силу следствия и отсутствия нулей у $B_{1} \in \mathcal{B}^{\prime \prime}$ отображение $\lambda_{I}: \mathcal{B}^{\prime \prime} \rightarrow C(Q)$ непрерывно в смысле $C^{1}$-топологии на $\mathcal{B}^{\prime \prime}$ и $C^{0}$-топологии на $C(Q)$. Значит, $\lambda_{I}(B)(w)$ и $\lambda_{I}(B)\left(w^{\prime}\right)$ суть пределы для $\left\{\lambda_{I}\left(B_{n}^{s}\right)\left(w_{n}\right)=\lambda_{I}\left(B_{n}^{s}\right)\left(w_{n}^{\prime}\right)\right\}$, а потому совпадают, откуда $\lambda_{I}(B)=$ const.

Имеем

$$
D_{B} I\left(A^{\prime}\right)=\lambda_{I}(B) \int_{Q} B \wedge A^{\prime}=\lambda_{I}(B) D_{B} \mathcal{H}_{\varkappa \perp}\left(A^{\prime}\right) .
$$

Значит, функционал $\left.I\right|_{\mathcal{B}^{\prime \prime}}$ локально постоянен на любом множестве уровня спиральности.

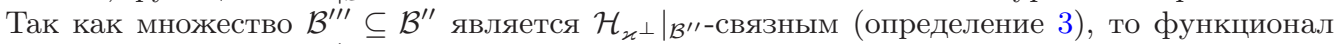
$\left.I\right|_{\mathcal{B}^{\prime \prime \prime}}$ постоянен на $\mathcal{H}_{\varkappa \perp}^{-1}(c) \cap \mathcal{B}^{\prime \prime \prime}$, т.е. равен некоторой константе $h(c)$, зависящей только от $c \in \mathbb{R}$ (для некоторой функции $h: \mathbb{R} \rightarrow \mathbb{R}$ ). Таким образом, $I(B)=h\left(\mathcal{H}_{\varkappa} \perp(B)\right)$ для любого $B \in \mathcal{B}^{\prime \prime \prime}$. Теорема доказана.

Ключевой результат [7] не обобщается на случай $C^{4}$-топологии [8].

Автор выражает благодарность Г. Хорнигу за постановку задачи, Л. В. Полтеровичу и Д. Пералта-Саласу за интерес и указание работ [9] и [7].

\section{СПИСОК ЦИТИРОВАННОЙ ЛИТЕРАТУРЫ}

[1] G. R. Jensen, Indiana Univ. Math. J., 20:12 (1971), 1125-1143. [2] A. Banyaga, Comment. Math. Helv., 53:2 (1978), 174-227. [3] E. Calabi, Problems in Analysis, Princeton Univ. Press, Princeton, NJ, 1970, 1-26. [4] Е. А. Кудрявцева, Матем. заметки, 95:6 (2014), 951-954. [5] E. Kudryavtseva, Proc. Int. Conf. "Knots and links in fluid flows: from helicity to knot energy", IUM Publications, Moscow, 2015, 9-10. [6] D. Serre, C. R. Acad. Sci. Paris Sér. A, 289:4 (1979), 267-270; Phys. D, 13:1-2 (1984), 105-136. [7] M. Bessa, C. R. Math. Acad. Sci. Paris, 346:21-22 (2008), 1169-1174. [8] А. Г. Медведев, Матем. заметки, 95:2 (2014), 227-233. [9] C. Bonatti, S. Crovisier, Invent. Math., 158:1 (2004), 33-104.

\section{Е. А. Кудрявцева}

Поступило

Московский государственный 10.11 .2015 университет имени М.В.Ломоносова

E-mail: eakudr@mech.math.msu.su 